\title{
AMathematical Mystery Tour: Discovering the Truth and Beauty of the Cosmos
}

Reviewed by Lynn Arthur Steen

\author{
A Mathematical Mystery Tour: Discovering the \\ Truth and Beauty of the Cosmos \\ A. K. Dewdney \\ John Wiley \& Sons, 1999 \\ ISBN 0-471-23847-3 \\ 224 pages, $\$ 22.95$
}

Debate about whether mathematics is invented or discovered has endured, emerging unresolved from every development in mathematics, philosophy, and science. Although most practicing mathematicians find this controversy irrelevant to their work-a preoccupation of philosophers who worry about distinctions without a difference-many of the world's greatest mathematicians have joined the fray. Yet despite remarkable agreement on questions of mathematical truth, on this one topic that probes the very nature of their discipline, mathematicians seem unable to agree.

The witnesses for mathematics as an invention or creation of the human mind include Augustus de Morgan ("The moving power of mathematical invention is not reasoning but imagination"), Janos Bolyai ("Out of nothing I have created a strange new universe"), David Hilbert ("Nothing will drive us out of the paradise that Cantor has created"), Albert Einstein ("The series of integers is obviously an invention of the human mind, a self-created tool which simplifies the ordering of certain sensory experiences"), and George Pólya ("If the learning of mathematics reflects to any degree the invention of mathematics, it must have a place for guessing,

Lynn Arthur Steen is professor of mathematics at St. Olaf College, Northfield, MN. His e-mail address is steen@ stolaf.edu.

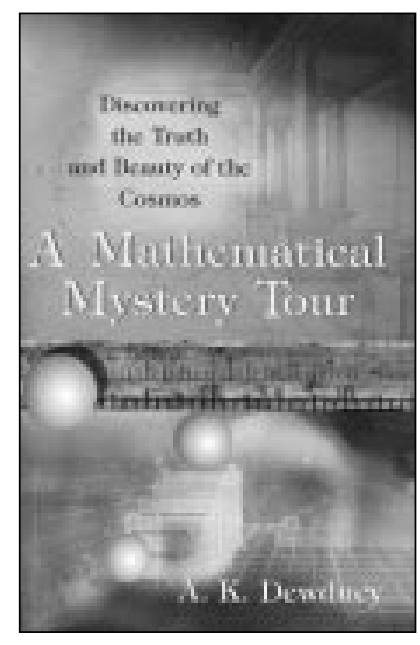

for plausible inference").

Speaking for mathematics as the discovery of objects and truths in a Platonic universe of ideals are Archimedes ("Once the method [for calculating the volume of a sphere] is understood and established, it will be used to discover other theorems which have not yet occurred to me, by other mathematicians, now living or yet unborn"), Isaac Newton ("I seem to have been only a boy playing on the sea-shore... whilst the great ocean of truth lay all undiscovered before me"), Leonhard Euler ("Mathematicians have tried in vain to this day to discover some order in the sequence of prime numbers, and we have reason to believe that it is a mystery into which the human mind will never penetrate"), and G. H. Hardy ("I believe that mathematical reality lies outside of us and that our function is to discover or observe it, and that the theorems which we... describe grandiloquently as our 'creations' are simply notes on our observations").

This debate is of more than academic interest, since it bears directly on a second major question about the nature of mathematics: How is it that mathematics is so incredibly useful in describing the natural world? As modern physics develops a 
"theory of everything" from increasingly abstract mathematics, as financial markets succumb to the magic of mathematical measures of risk, and as biology employs combinatorial algorithms to unlock the genetic code, it does not seem far-fetched to imagine, as the Pythagoreans once did, that numbers hold the key to the universe.

As a cult the Pythagoreans had a good run, from about 500 B.C.E. until well into the Islamic era. Common wisdom holds that theirs was a pre-scientific belief, a close cousin of astrology and numerology, rendered obsolete by the rise of science that provided more effective explanations of natural events. But science has now come virtually full circle, restoring mathematics to a throne not unlike that imagined by the ancient Pythagoreans.

Whether we recognize it or not, the information age in which we live confronts us once again with the ancient mystery of why the universe is so mathematical. Is the effectiveness of mathematics truly "unreasonable", as Eugene Wigner [7] famously proclaimed, a miraculous gift which we "neither understand nor deserve"? Or is it tautological, as David Lindley [4] has argued, because "we reserve the name 'science' for anything that mathematics can handle"? Or is it, as the Pythagoreans believed, because the world is actually made of mathematics? Put more bluntly: Does the cosmos make mathematics, or does mathematics make the cosmos?

It is this mystery that A. K. (Alexander Keewatin) Dewdney sets out to solve in A Mathematical Mystery Tour. But instead of offering a scholarly tome fortified with footnotes and references, computer scientist Dewdney-author of several popular books on science, mathematics, and computer science-cloaks his argument in a Socratic docudrama in which the author explores the development of mathematics with contemporary fictional experts representing four major sites and epochs-ancient Greece, early Islam, post-Renaissance Italy, and twentieth-century England.

Dewdney begins in Miletus, near the ancient home of Pythagoras, where the fictional Dr. Petros Pygonopolis explains to straight-man Dewdney the intellectual challenge of incommensurables that his Greek progenitors had overcome (they called these numbers a-logos or "illogical") as well as how they used the gnomon (carpenter's square) to prove what we now call the Pythagorean Theorem. "Rest assured," comments the fictional professor, "if Pythagoras had not discovered the famous theorem named after him, someone else would have."

The two protagonists, the author and his fictional tutor, discuss with some excitement the irony that modern computers inhabit a Pythagorean world devoid of irrational numbers. "Imagine," exclaims Pygonopolis, "the world of Pythagoras lives again in computers. What a wonderful idea!"
The neo-Pythagorean Pygonopolis then introduces Dewdney to the holos, the place where (Platonic) mathematics lives. "Holos stands in special relation to the cosmos" he explains. "Holos is the source, cosmos the manifestation." For the remainder of his tour, Dewdney will inquire about the holos and seek to uncover clues about its relation to the cosmos.

Dewdney then travels to the Arabian desert seeking insight into ancient Islamic arithmetic and astronomy under the tutelage of Professor AlFlayli. Together they explore the similarities and differences among the various number systems that were the subject of lively debate in Baghdad around 800-1000 C.E. The differences in notation are, according to Al-Flayli, "cultural and invented," but the similarity of meaning goes "beyond culture." "I would maintain," he is reported to have said, "that it was discovered."

Al-Flayli goes on to explain to the inquiring Dewdney how in Islamic culture numbers occupy a "Superior World," a place somewhat akin to the Greek holos. "The human mind creates numbers in the same sense that it creates colors. Yet the colors we perceive correspond to something real outside the mind." In this sense, "we are discovering numbers all the time."

Their discussion provides an excuse for Dewdney to introduce ideas about amicable numbers (as an illustration of how the cultural fascination with the "personality" of numbers influenced Islamic scholars), symmetry groups (well represented in Islamic art), the astrolabe (an Islamic instrument of trigonometry), and astronomy (notably the celestial sphere with its puzzling retrograde planetary motions). The latter struggle between the Ptolemaic and Copernican theories leads to much dialogue about the empirically verifiable nature of science. "Paradoxical as it may sound," intones Al-Flayli, "only the possibility of being wrong will save science from becoming a purely cultural exercise."

Like a good student, before alighting at his next stop Dewdney reflects on the lessons so far learned. Two examples of cultural beliefs foundered on the rock of mathematical reality: the Greek belief that all distances are commensurable and the widespread pre-Copernican belief that the stars reside on the celestial sphere. His next conjured host, science historian Maria Canzoni in Venice, adds more evidence. She explains how the nineteenthcentury Swiss mathematician Johann Jacob Balmer used an essentially Pythagorean method (searching for ratios of integers) to find an extremely accurate two-variable formula for the spectral lines of hydrogen, which at that time had only recently been discovered.

The meaning of Balmer's formula became clear only decades later when Niels Bohr introduced the quantum theory of the atom that had at its very foundation the idea that energy, like number, is 
discrete. As Canzoni tells it, the Pythagoreans' integer theory of the cosmos was reborn in quantum theory. "Balmer's discovery is a particularly striking example of mathematical patterns that reside in nearly every aspect of physical reality. I believe that these [patterns] are there because something within the cosmos satisfies the axioms within the holos."

To clinch her argument, Canzoni goes on to educate tourist Dewdney on the elements of calculus in order to explain the reasoning that led to the discovery of Neptune. This leads naturally (and very briefly) to a discussion of special relativity and the quantum model of the atom in order to illustrate the equivalence of matter (cosmos) with energy, and of energy-through the Schrödinger equationwith information (holos). "You could say," exclaims Canzoni, "that this equation is the hydrogen atom."

Of course, the equations themselves-and others yet to be discovered-are neither energy nor matter. They are what Canzoni calls "information systems" that inhabit the holos. In her theory (which may or may not be Dewdney's view-one can never be sure with these fictional dialogues) the "truth intersections" of these holos-inhabitants manifest themselves as atoms in the cosmos. Undaunted by the audacity of this suggestion, she goes on to claim that what is missing from physics is a theory of mind, a menos that connects the holos to the cosmos. (Canzoni's suggestion echoes many of the speculations advanced a decade ago by Roger Penrose in seeking a scientifically respectable theory that unifies physics, computation, and consciousness [5].)

On to Sir John Brainard at Merton College, Oxford, who dismisses the holos as an "old chestnut." Brainard (aka Dewdney) uses a minilecture on group theory dressed up in new language as a launching point for a discussion of the mechanization of mathematics, "the greatest development, in my humble view, of twentieth century mathematics." (Dewdney is, after all, a computer scientist.) This leads naturally to the oft-told story of Hilbert's belief, Gödel's theorem, Turing's machine, Church's thesis, and, finally, "mind machines" or "engines of thought." Bits of information-0's and 1's-existing independently of media or form constitute the essence of mathematics. "Computers establish at least one thing about mathematical realities," concludes Brainard. "They no longer depend on the mind of man, and the evanescence of their existence is the very proof of their ultimate reality."

Reflecting on his information-filled tutorial in the company of fictional experts, Dewdney takes some parting shots at culturally based fads such as postmodernism and paradigm shifts. Instead, he latches onto the vague (and very Platonic) idea of "essential content" that transcends every culturebased attempt at description. The essential content of (computable) mathematics can be found in the binary digits of computers; the essential content of living organisms can be found in the coded language of DNA, which persists in descendents after each organism's death.

In the end-perhaps also from the beginningDewdney sides with those who say that mathematics is discovered. But he goes much further, suggesting that perhaps Pythagoras was right after all. "Is the cosmos made of number? One can scarcely entertain such an outlandish proposition." Yet for Dewdney the answer is inescapable: "The holos is where mathematics exists. Its landmarks of essential content, from numbers to theorems, persist like geographical features. Indeed, they have a permanent existence. The essential content of mathematics is not created; it is discovered."

And why is the cosmos built to reflect the axioms of the holos? "Perhaps there is no other way for a cosmos to be structured. The cosmos exists because there is a mind that can think it." In other words, mathematics (holos) is the real reality, while the world (cosmos) is just an invention of our minds.

Dewdney's fictional guides add drama to what might otherwise be a bland intellectual argument. (There do remain, however, lengthy passages of mathematical exposition that will probably exceed the patience both of readers who suffer from math anxiety and of those who think of themselves as mathematical cognoscenti.) But entertaining exposition is no substitute for evidence. Dewdney argues his case entirely on the basis of personal speculation anchored in his selective account of the history of mathematics. He provides no references to document any of the mathematical developments described in the narratives of his fictional protagonists, leaving the reader with no means of verifying his claims nor any invitation to further reading.

Even more disappointing than the lack of documentation is the omission of any reference to contemporary scientific discoveries. Recent research in neuroscience has provided significant new evidence about the evolutionary and physiological connection between mind and mathematics (e.g., [1], [2], [3], [6]), evidence that was not available to any of the giants in the history of mathematics whose ideas form the centerpiece of Dewdney's analysis. This new evidence, which Dewdney totally ignores, changes the terms of the debate about the mystery of mathematics as much as the discovery of germs changed the debate about the mystery of disease.

The issue is no longer only one of discovery or invention, but also now of evolution. Mounting evidence suggests that the rudiments of arithmetic are "anchored in our genes" [3, p. 249], that infants are born with a capacity for recognizing and distinguishing among small numerosities [1, p. 146], 
and that mathematical objects are the "residue" (a Darwinian term) of objects that have been "selected for their fitness and their coherence" [2, p. 36]. According to this evolutionary hypothesis, natural selection has acted through phylogenetic evolution to ensure that the brain constructs internal representations that are "advantageously adapted to the regularities of the universe" [3, p. 249].

Mathematics may well be one such adaptation. The supposed "miracle" of the unreasonable effectiveness of mathematics in the natural world can be accounted for as easily by selective evolution as can the "miracle" of the adaptation of the eye to sight. Although the structures of the cosmos certainly predate the human mind, they are not necessarily inherited from the "truth-intersections" of a preexistent holos. The evidence from biology suggests an important alternative hypothesis: that the human brain, equipped by evolution, translates these structures into mathematics.

Whereas Dewdney posits a preexisting holos as the only possible explanation for features of mathematics that transcend human culture, many neuroscientists now say that genes offer a more plausible explanation. Whereas Dewdney argues from logic and history in favor of the Pythagorean belief that numbers control the universe, biologists claim to explain the same facts as adaptation based on natural selection. One wonders why Dewdney, who held a joint appointment in zoology, ignored this alternative hypothesis. Maybe he just got stuck with the wrong tour guides.

\section{References}

[1] Brian BUTTERWORTH, What Counts: How Every Brain Is Hardwired for Math, The Free Press, New York, 1999.

[2] Jean-Pierre Changeux and Alain Connes, Conversations on Mind, Matter, and Mathematics, Princeton University Press, Princeton, NJ, 1995.

[3] Stanislas Dehaene, The Number Sense: How the Mind Creates Mathematics, Oxford University Press, New York, 1997.

[4] DAVID LINDLEY, The End of Physics: The Myth of a Unified Theory, Basic Books, New York, 1994.

[5] Roger Penrose, The Emperor's New Mind: Concerning Computers, Minds, and the Laws of Physics, Oxford University Press, New York, 1989.

[6] STEVEn PinKer, How the Mind Works, W. W. Norton, New York, 1997.

[7] EugENE WignER, The unreasonable effectiveness of mathematics, Comm. Pure Appl. Math. 13 (1960), $1-4$. 